\title{
Hydrogen Bonds of RNA Are Stronger than Those of DNA, But NMR Monitors Only Presence of Methyl Substituent in Uracil/Thymine.
}

\author{
Marcel Swart, Célia Fonseca Guerra, and F. Matthias Bickelhaupt* \\ Contribution from the Afdeling Theoretische Chemie, Scheikundig Laboratorium der Vrije Universiteit, \\ De Boelelaan 1083, NL-1081 HV Amsterdam, The Netherlands. E-mail: F.M.Bickelhaupt@few.vu.nl
}

\section{Contents:}

- NMR shielding versus isotope effect

- Atomic coordinates base pairs

- Table S1. Results using TZ2P basis set

- Table S2. ZORA relativistic results (QZ4P basis)

- $\quad$ Table S3. A: $\mathrm{U}^{\mathrm{Me} 6}, \mathrm{~A}^{\mathrm{Me}}: \mathrm{U}$ and displaced base pairs

- Table S4. Solvent effects and Hartree-Fock data

- Figure S1. Influence of basis set cq. DFT functional on NMR shielding C2

- Definition of coordinate axes for variations of base pair geometry

- Figure S2. Variation of base pair geometry: Stretch

- Figure S3. Variation of base pair geometry: Shear

- Figure S4. Variation of base pair geometry: Propellor Twist

- Figure S5. Variation of base pair geometry: Buckle 


\section{NMR shielding versus isotope effect}

The experimental evidence consists of changes in deuterium isotope effects, which are defined as:

$$
\Delta^{13} \mathrm{C} 2=\delta^{13} \mathrm{C} 2\left\{{ }^{1} \mathrm{H} 3\right\}-\delta^{13} \mathrm{C} 2\left\{{ }^{2} \mathrm{H} 3\right\}
$$

$\mathrm{H} / \mathrm{D}$ isotope substitution affects only the average $\mathrm{N}-{ }^{1,2} \mathrm{H}$ distance which, in turn, influences the chemical shift linearly. We have tested this explicitly in a numerical experiment with an A:T (A:U) base pair designated "A:T H3 displaced" (“A:U H3 displaced”), in which we have placed the H3 atom of the pyrimidine base at a distance from $\mathrm{N} 3$ that it has in $\mathrm{A}: \mathrm{U}(\mathrm{A}: \mathrm{T})$. This simulates a change in average $\mathrm{N} 3-\mathrm{H} 3$ distance. Thus, we compute adenine C2 chemical shielding constants of 32.438 (A:T), 32.445 (A:T H3 displ.), 32.538 (A:U) and 32.531 ppm (A:U H3 displ.). The resulting change in chemical shift of adenine $\mathrm{C} 2$ appears to be proportional to the chemical shift: $\delta^{13} \mathrm{C} 2\left\{{ }^{A T H 3}{ }^{d i s p l} \mathrm{H} 3\right\} / \delta^{13} \mathrm{C} 2\left\{{ }^{A T} \mathrm{H} 3\right\}=1.000216$ and $\delta^{13} \mathrm{C} 2\left\{{ }^{A U} \mathrm{H} 3\right\} / \delta^{13} \mathrm{C} 2\left\{{ }^{A U H 3}{ }^{d i s p l} \mathrm{H} 3\right\}=1.000215$. Note that the change in $\mathrm{N} 3-\mathrm{H} 3$ distance we use in this simulation is not equal to the change in average $\mathrm{N} 3{ }^{1,2} \mathrm{H} 3$ distance due to the H/D isotope substitution but, within this regime of changes in (average) bond lengths, the linear behavior should not be influenced by the actual step size. Thus, we find the change in chemical shift due to the H/D isotope effect to be proportional to the chemical shift itself: $\delta^{13} \mathrm{C} 2\left\{{ }^{1} \mathrm{H} 3\right\}-\delta^{13} \mathrm{C} 2\left\{{ }^{2} \mathrm{H} 3\right\}=f \cdot \delta^{13} \mathrm{C} 2\left\{{ }^{1} \mathrm{H} 3\right\}$. Therefore, the experimentally observed H/D isotope effect results from the larger chemical shift of adenine $\mathrm{C} 2$ in A:U vs. A:T: $\Delta^{13} \mathrm{C} 2_{R N A}-\Delta^{13} \mathrm{C} 2_{D N A}=f\left(\delta^{13} \mathrm{C} 2_{R N A}-\delta^{13} \mathrm{C} 2_{D N A}\right)$. 
Atomic Cartesian coordinates (̊̊)

$\begin{array}{lrrr} & \text { A:T } & & \\ \text { N } & -2.804619 & 2.870483 & 0.000000 \\ \mathrm{C} & -1.500876 & 3.214866 & 0.000000 \\ \mathrm{~N} & -0.436204 & 2.409711 & 0.000000 \\ \mathrm{C} & -0.804028 & 1.117367 & 0.000000 \\ \mathrm{C} & -2.109100 & 0.608355 & 0.000000 \\ \mathrm{C} & -3.155707 & 1.559337 & 0.000000 \\ \mathrm{~N} & 0.016004 & 0.006202 & 0.000000 \\ \mathrm{C} & -0.828525 & -1.087949 & 0.000000 \\ \mathrm{~N} & -2.108613 & -0.774157 & 0.000000 \\ \mathrm{~N} & -4.456964 & 1.227537 & 0.000000 \\ \mathrm{H} & -1.312320 & 4.289149 & 0.000000 \\ \mathrm{C} & 1.471397 & 0.024194 & 0.000000 \\ \mathrm{H} & -0.434849 & -2.099777 & 0.000000 \\ \mathrm{H} & -4.704639 & 0.247632 & 0.000000 \\ \mathrm{H} & -5.185612 & 1.959427 & 0.000000 \\ \mathrm{~N} & -4.723472 & 4.924934 & 0.000000 \\ \mathrm{C} & -4.220413 & 6.210505 & 0.000000 \\ \mathrm{~N} & -5.204958 & 7.213582 & 0.000000 \\ \mathrm{C} & -6.545550 & 6.899802 & 0.000000 \\ \mathrm{C} & -7.027132 & 5.629209 & 0.000000 \\ \mathrm{C} & -6.057292 & 4.540684 & 0.000000 \\ \mathrm{O} & -6.374784 & 3.337976 & 0.000000 \\ \mathrm{O} & -3.018553 & 6.460244 & 0.000000 \\ \mathrm{C} & -8.488872 & 5.291927 & 0.000000 \\ \mathrm{H} & -3.991273 & 4.149302 & 0.000000 \\ \mathrm{C} & -4.774321 & 8.612272 & 0.000000 \\ \mathrm{H} & -7.213616 & 7.761549 & 0.000000 \\ \mathrm{H} & -8.749389 & 4.687478 & 0.879911 \\ \mathrm{H} & -9.106892 & 6.198386 & 0.000000 \\ \mathrm{H} & -8.749389 & 4.687478 & -0.879911 \\ \mathrm{H} & -5.143900 & 9.126706 & 0.896477 \\ \mathrm{H} & -3.681447 & 8.616543 & 0.000000 \\ \mathrm{H} & -5.143900 & 9.126706 & -0.896477 \\ \mathrm{H} & 1.866121 & -0.471407 & -0.895642 \\ \mathrm{H} & 1.786238 & 1.072518 & 0.000000 \\ \mathrm{H} & 1.866121 & -0.471407 & 0.895642\end{array}$

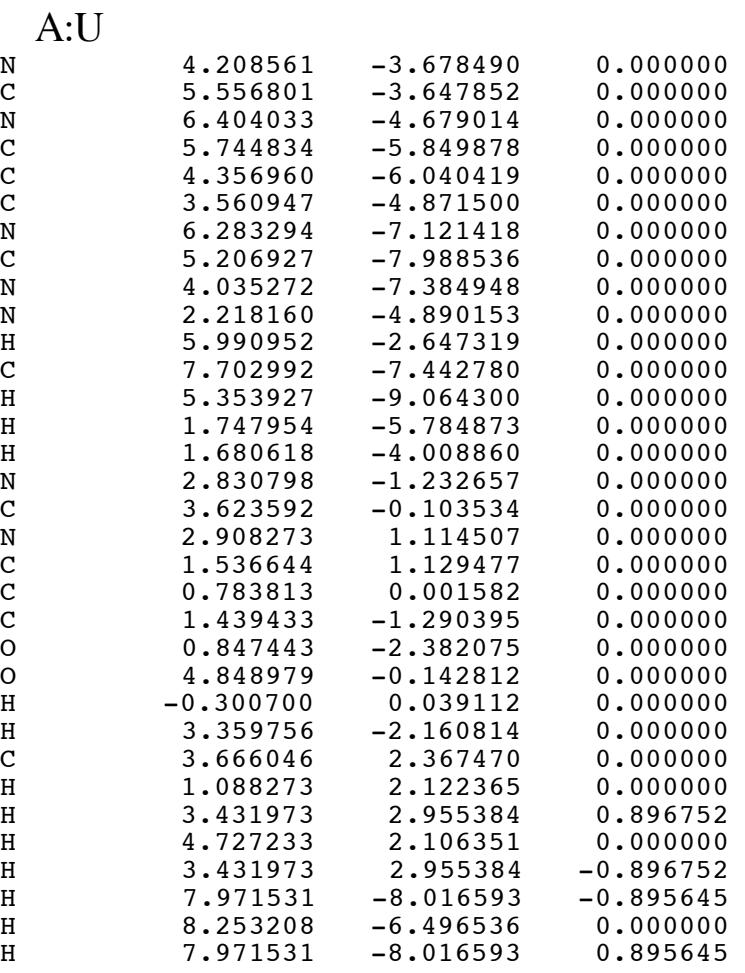
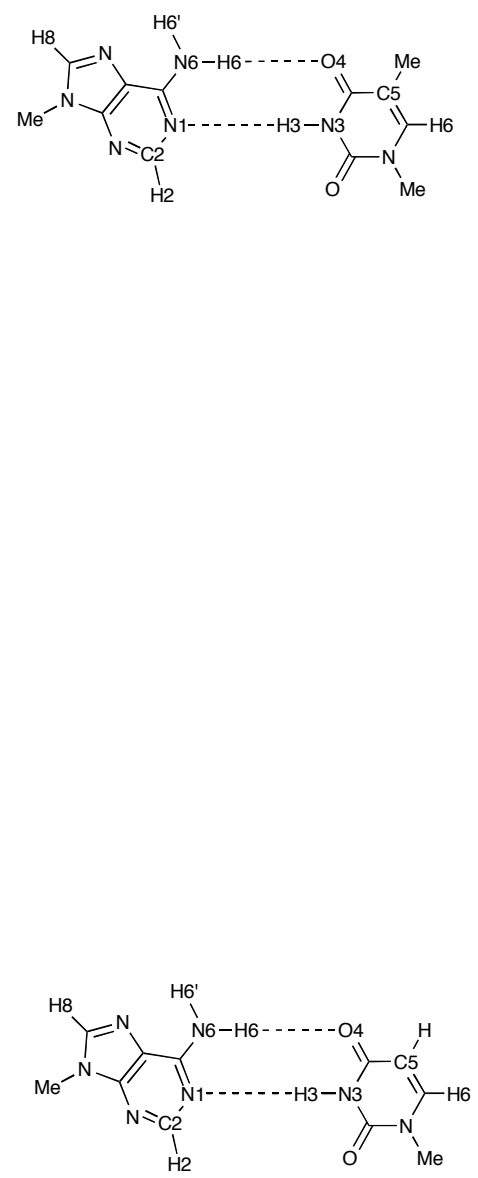
Atomic Cartesian coordinates (̊̊) continued

\begin{tabular}{|c|c|c|c|}
\hline $\mathrm{N}$ & -3.549974 & -3.559427 & 0.000000 \\
\hline C & -4.896348 & -3.482178 & 0.000000 \\
\hline $\mathrm{N}$ & -5.778735 & -4.483422 & 0.000000 \\
\hline C & -5.160424 & -5.676384 & 0.000000 \\
\hline C & -3.779970 & -5.914810 & 0.000000 \\
\hline C & -2.944007 & -4.774120 & 0.000000 \\
\hline $\mathrm{N}$ & -5.742538 & -6.928541 & 0.000000 \\
\hline C & -4.696804 & -7.832366 & 0.000000 \\
\hline $\mathrm{N}$ & -3.504975 & -7.269660 & 0.000000 \\
\hline $\mathrm{N}$ & -1.602668 & -4.839202 & 0.000000 \\
\hline $\mathrm{H}$ & -5.295636 & -2.467229 & 0.000000 \\
\hline C & -7.172501 & -7.200611 & 0.000000 \\
\hline $\mathrm{H}$ & -4.880921 & -8.902403 & 0.000000 \\
\hline $\mathrm{H}$ & -1.163687 & -5.749649 & 0.000000 \\
\hline $\mathrm{H}$ & -1.034969 & -3.977027 & 0.000000 \\
\hline $\mathrm{N}$ & -2.088447 & -1.162706 & 0.000000 \\
\hline $\mathrm{C}$ & -2.841716 & -0.006840 & 0.000000 \\
\hline $\mathrm{N}$ & -2.084699 & 1.185733 & 0.000000 \\
\hline C & -0.713373 & 1.153257 & 0.000000 \\
\hline C & 0.000000 & 0.000000 & 0.000000 \\
\hline C & -0.699911 & -1.268530 & 0.000000 \\
\hline 0 & -0.146030 & -2.380030 & 0.000000 \\
\hline $\mathrm{O}$ & -4.067728 & -0.003715 & 0.000000 \\
\hline C & 1.503511 & -0.026989 & 0.000000 \\
\hline $\mathrm{H}$ & -2.649188 & -2.072014 & 0.000000 \\
\hline $\mathrm{C}$ & -2.798686 & 2.464154 & 0.000000 \\
\hline $\mathrm{H}$ & -0.230932 & 2.130044 & 0.000000 \\
\hline $\mathrm{H}$ & -2.544420 & 3.043621 & 0.896752 \\
\hline $\mathrm{H}$ & -3.868268 & 2.239892 & 0.000000 \\
\hline $\mathrm{H}$ & -2.544420 & 3.043621 & -0.896752 \\
\hline $\mathrm{H}$ & -7.460725 & -7.764793 & -0.895645 \\
\hline $\mathrm{H}$ & -7.689662 & -6.235904 & 0.000000 \\
\hline $\mathrm{H}$ & -7.460725 & -7.764793 & 0.895645 \\
\hline $\mathrm{H}$ & 1.884142 & -0.564329 & -0.879742 \\
\hline $\mathrm{H}$ & 1.921663 & 0.987273 & 0.000000 \\
\hline $\mathrm{H}$ & 1.884142 & -0.564329 & 0.879742 \\
\hline
\end{tabular}

A:U//A:T

\begin{tabular}{|c|c|c|c|}
\hline $\mathrm{N}$ & 4.244228 & 3.637454 & 0.000000 \\
\hline $\mathrm{C}$ & 5.592021 & 3.595012 & 0.000000 \\
\hline $\mathrm{N}$ & 6.448409 & 4.618926 & 0.000000 \\
\hline $\mathrm{C}$ & 5.799441 & 5.795483 & 0.000000 \\
\hline $\mathrm{C}$ & 4.413340 & 5.998041 & 0.000000 \\
\hline $\mathrm{C}$ & 3.607341 & 4.836095 & 0.000000 \\
\hline $\mathrm{N}$ & 6.348652 & 7.062570 & 0.000000 \\
\hline $\mathrm{C}$ & 5.279745 & 7.938830 & 0.000000 \\
\hline $\mathrm{N}$ & 4.102981 & 7.345266 & 0.000000 \\
\hline $\mathrm{N}$ & 2.264800 & 4.866835 & 0.000000 \\
\hline $\mathrm{H}$ & 6.017283 & 2.590627 & 0.000000 \\
\hline C & 7.770829 & 7.372258 & 0.000000 \\
\hline $\mathrm{H}$ & 5.435850 & 9.013264 & 0.000000 \\
\hline $\mathrm{H}$ & 1.803151 & 5.765967 & 0.000000 \\
\hline $\mathrm{H}$ & 1.719360 & 3.989860 & 0.000000 \\
\hline $\mathrm{N}$ & 2.836410 & 1.204181 & 0.000000 \\
\hline $\mathrm{C}$ & 3.615628 & 0.064628 & 0.000000 \\
\hline $\mathrm{N}$ & 2.881814 & -1.134125 & 0.000000 \\
\hline $\mathrm{C}$ & 1.504997 & -1.129787 & 0.000000 \\
\hline $\mathrm{C}$ & 0.750074 & 0.000000 & 0.000000 \\
\hline $\mathrm{C}$ & 1.450348 & 1.278708 & 0.000000 \\
\hline 0 & 0.870576 & 2.379241 & 0.000000 \\
\hline 0 & 4.842867 & 0.091501 & 0.000000 \\
\hline $\mathrm{H}$ & -0.333865 & -0.018084 & 0.000000 \\
\hline $\mathrm{H}$ & 3.375476 & 2.124577 & 0.000000 \\
\hline C & 3.615897 & -2.400184 & 0.000000 \\
\hline $\mathrm{H}$ & 1.047784 & -2.119674 & 0.000000 \\
\hline $\mathrm{H}$ & 3. 371441 & -2.984540 & -0.896477 \\
\hline $\mathrm{H}$ & 4.681751 & -2.158632 & 0.000000 \\
\hline $\mathrm{H}$ & 3.371441 & -2.984540 & 0.896477 \\
\hline $\mathrm{H}$ & 8.044020 & 7.943917 & 0.895642 \\
\hline $\mathrm{H}$ & 8.313306 & 6.421560 & 0.000000 \\
\hline $\mathrm{H}$ & 8.044020 & 7.943917 & -0.895642 \\
\hline
\end{tabular}
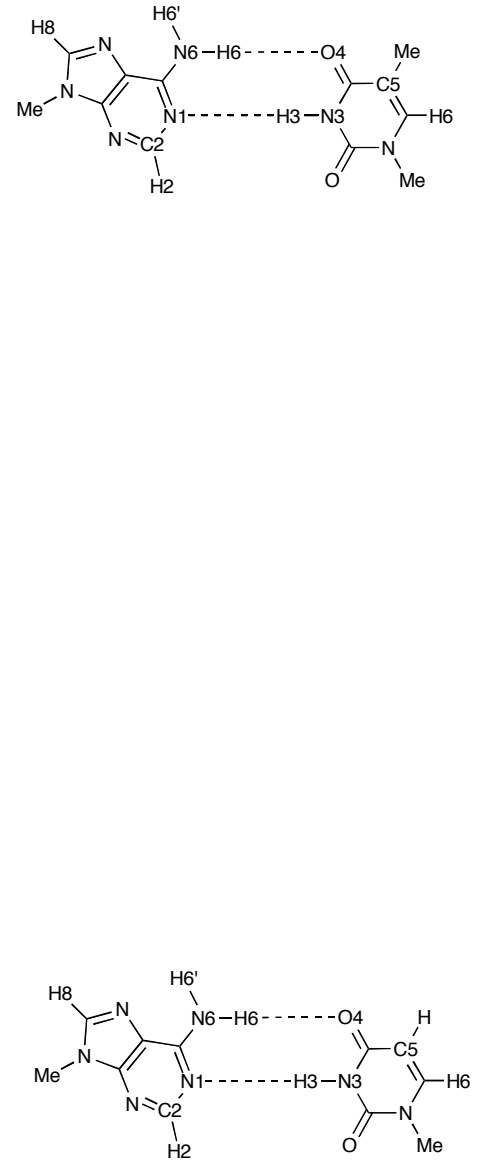
Atomic Cartesian coordinates $(\AA)$ continued

$\begin{array}{lrrr} & \text { A:T } & & \\ \mathrm{N} & 2.75039 & -2.32710 & 0.00000 \\ \mathrm{C} & 3.37555 & -3.52195 & 0.00000 \\ \mathrm{~N} & 4.68772 & -3.76621 & 0.00000 \\ \mathrm{C} & 5.39189 & -2.62274 & 0.00000 \\ \mathrm{C} & 4.88888 & -1.31564 & 0.00000 \\ \mathrm{C} & 3.48050 & -1.18344 & 0.00000 \\ \mathrm{~N} & 6.76126 & -2.46285 & 0.00000 \\ \mathrm{C} & 7.00648 & -1.10412 & 0.00000 \\ \mathrm{~N} & 5.90878 & -0.37908 & 0.00000 \\ \mathrm{~N} & 2.85002 & 0.00142 & 0.00000 \\ \mathrm{H} & 2.71044 & -4.38645 & 0.00000 \\ \mathrm{H} & 7.44291 & -3.21216 & 0.00000 \\ \mathrm{H} & 8.01709 & -0.71046 & 0.00000 \\ \mathrm{H} & 3.40748 & 0.84466 & 0.00000 \\ \mathrm{H} & 1.81845 & 0.04774 & 0.00000 \\ \mathrm{~N} & -0.05701 & -2.28833 & 0.00000 \\ \mathrm{C} & -0.67704 & -3.52147 & 0.00000 \\ \mathrm{~N} & -2.07067 & -3.43857 & 0.00000 \\ \mathrm{C} & -2.76521 & -2.25240 & 0.00000 \\ \mathrm{C} & -2.13984 & -1.04792 & 0.00000 \\ \mathrm{C} & -0.67692 & -1.04295 & 0.00000 \\ \mathrm{O} & -0.00181 & 0.00013 & 0.00000 \\ \mathrm{O} & -0.08692 & -4.59487 & 0.00000 \\ \mathrm{C} & -2.86066 & 0.26727 & 0.00000 \\ \mathrm{H} & 1.01032 & -2.30578 & 0.00000 \\ \mathrm{H} & -2.55357 & -4.32998 & 0.00000 \\ \mathrm{H} & -3.85016 & -2.34630 & 0.00000 \\ \mathrm{H} & -2.58306 & 0.86358 & 0.88018 \\ \mathrm{H} & -3.94834 & 0.12396 & 0.00000 \\ \mathrm{H} & -2.58306 & 0.86358 & -0.88018 \\ & & & \end{array}$

\section{A:U}

$\begin{array}{lrrr}\text { N } & 2.74845 & -2.32276 & 0.00000 \\ \mathrm{C} & 3.37110 & -3.51911 & 0.00000 \\ \mathrm{~N} & 4.68248 & -3.76608 & 0.00000 \\ \mathrm{C} & 5.38916 & -2.62415 & 0.00000 \\ \mathrm{C} & 4.88902 & -1.31590 & 0.00000 \\ \mathrm{C} & 3.48089 & -1.18048 & 0.00000 \\ \mathrm{~N} & 6.75875 & -2.46750 & 0.00000 \\ \mathrm{C} & 7.00709 & -1.10931 & 0.00000 \\ \mathrm{~N} & 5.91105 & -0.38179 & 0.00000 \\ \mathrm{~N} & 2.85293 & 0.00571 & 0.00000 \\ \mathrm{H} & 2.70422 & -4.38223 & 0.00000 \\ \mathrm{H} & 7.43861 & -3.21848 & 0.00000 \\ \mathrm{H} & 8.01864 & -0.71801 & 0.00000 \\ \mathrm{H} & 3.41190 & 0.84800 & 0.00000 \\ \mathrm{H} & 1.82197 & 0.05488 & 0.00000 \\ \mathrm{~N} & -0.05409 & -2.28295 & 0.00000 \\ \mathrm{C} & -0.67240 & -3.51596 & 0.00000 \\ \mathrm{~N} & -2.07292 & -3.44273 & 0.00000 \\ \mathrm{C} & -2.76908 & -2.26297 & 0.00000 \\ \mathrm{C} & -2.13059 & -1.06848 & 0.00000 \\ \mathrm{C} & -0.67730 & -1.03457 & 0.00000 \\ \mathrm{O} & -0.00491 & 0.00785 & 0.00000 \\ \mathrm{O} & -0.08437 & -4.58894 & 0.00000 \\ \mathrm{H} & -2.67145 & -0.12782 & 0.00000 \\ \mathrm{H} & 1.01509 & -2.30035 & 0.00000 \\ \mathrm{H} & -2.54810 & -4.33868 & 0.00000 \\ \mathrm{H} & -3.85332 & -2.35428 & 0.00000\end{array}$


Atomic Cartesian coordinates $(\AA)$ continued

\begin{tabular}{|c|c|c|c|}
\hline \multicolumn{4}{|c|}{ A:U $\mathrm{U}^{\mathrm{Me} 6}$} \\
\hline $\mathrm{N}$ & -2.801177 & 2.871617 & 0.000000 \\
\hline & -1.497133 & 3.213587 & 0.000000 \\
\hline N & -0.433813 & 2.406737 & 0.000000 \\
\hline & -0.804415 & 1.115131 & 0.000000 \\
\hline C & -2.110347 & 0.608180 & 0.000000 \\
\hline & -3.155673 & 1.561105 & 0.000000 \\
\hline & 0.014089 & 0.002939 & 0.000000 \\
\hline & -0.831932 & -1.090058 & 0.000000 \\
\hline & -2.111598 & -0.774403 & 0.000000 \\
\hline & -4.457599 & 1.233282 & 0.000000 \\
\hline & -1.306610 & 4.287506 & 0.000000 \\
\hline & 1.469452 & 0.020063 & 0.000000 \\
\hline & -0.439600 & -2.102407 & 0.000000 \\
\hline & -4.707929 & 0.253993 & 0.000000 \\
\hline & -5.185269 & 1.967572 & 0.000000 \\
\hline & -4.717825 & 4.923261 & 0.000000 \\
\hline & -4.225799 & 6.207727 & 0.000000 \\
\hline & -5.207845 & 7.228494 & 0.000000 \\
\hline & -6.560655 & 6.933511 & 0.000000 \\
\hline & -6.991343 & 5.639136 & 0.000000 \\
\hline & -6.054343 & 4.541143 & 0.000000 \\
\hline & -6.372305 & 3.338753 & 0.000000 \\
\hline & -3.024562 & 6.459617 & 0.000000 \\
\hline & -7.523643 & 8.091664 & 0.000000 \\
\hline & -3.982596 & 4.150305 & 0.000000 \\
\hline & -4.718593 & 8.609746 & 0.000000 \\
\hline & -8.051503 & 5.404391 & 0.000000 \\
\hline & -7.384024 & 8.728719 & 0.886434 \\
\hline & -8.553589 & 7.717130 & 0.000000 \\
\hline & -7.384024 & 8.728719 & -0.886434 \\
\hline & -5.058484 & 9.145499 & 0.895403 \\
\hline & -3.627167 & 8.557542 & 0.000000 \\
\hline & -5.058484 & 9.145499 & -0.895403 \\
\hline & 1.864062 & -0.475581 & -0.895688 \\
\hline & 1.784619 & 1.068330 & 0.000000 \\
\hline & 1.864062 & -0.475581 & 0.895688 \\
\hline & 6//A:U & & \\
\hline & -5.481554 & 0.456349 & 0.000000 \\
\hline & -6.008518 & 1.697719 & 0.000000 \\
\hline & -7.296989 & 2.045481 & 0.000000 \\
\hline & -8.092790 & 0.962814 & 0.000000 \\
\hline & -7.695247 & -0.380488 & 0.000000 \\
\hline & -6.302306 & -0.624874 & 0.000000 \\
\hline & -9.473258 & 0.930236 & 0.000000 \\
\hline & -9.820539 & -0.407618 & 0.000000 \\
\hline & -8.788229 & -1.227027 & 0.000000 \\
\hline & -5.766663 & -1.856341 & 0.000000 \\
\hline & -5.275332 & 2.505179 & 0.000000 \\
\hline & -10.350437 & 2.091861 & 0.000000 \\
\hline & -10.861470 & -0.716391 & 0.000000 \\
\hline & -6.388574 & -2.653112 & 0.000000 \\
\hline & -4.742237 & -1.983538 & 0.000000 \\
\hline & -2.685431 & 0.207298 & 0.000000 \\
\hline & -1.982655 & 1.394542 & 0.000000 \\
\hline & -0.578156 & 1.243915 & 0.000000 \\
\hline & 0.000000 & 0.000000 & 0.000000 \\
\hline & -0.718104 & -1.150317 & 0.000000 \\
\hline & -2.165417 & -1.084528 & 0.000000 \\
\hline & -2.916711 & -2.073352 & 0.000000 \\
\hline & -2.522777 & 2.495171 & 0.000000 \\
\hline & -0.237553 & -2.123275 & 0.000000 \\
\hline & -3.749035 & 0.307385 & 0.000000 \\
\hline & 0.251898 & 2.450210 & 0.000000 \\
\hline & 1.512848 & -0.108636 & 0.000000 \\
\hline & 0.884048 & 2.478844 & 0.896752 \\
\hline & -0.422827 & 3.309888 & 0.000000 \\
\hline & 0.884048 & 2.478844 & -0.896752 \\
\hline & -10.983921 & 2.100442 & -0.895645 \\
\hline & -9.714498 & 2.982757 & 0.000000 \\
\hline & -10.983921 & 2.100442 & 0.895645 \\
\hline & 1.840430 & -0.671984 & -0.885168 \\
\hline & 2.035448 & 0.850364 & 0.000000 \\
\hline & 1.840430 & -0.671984 & 0.885168 \\
\hline
\end{tabular}


Atomic Cartesian coordinates $(\AA)$ continued

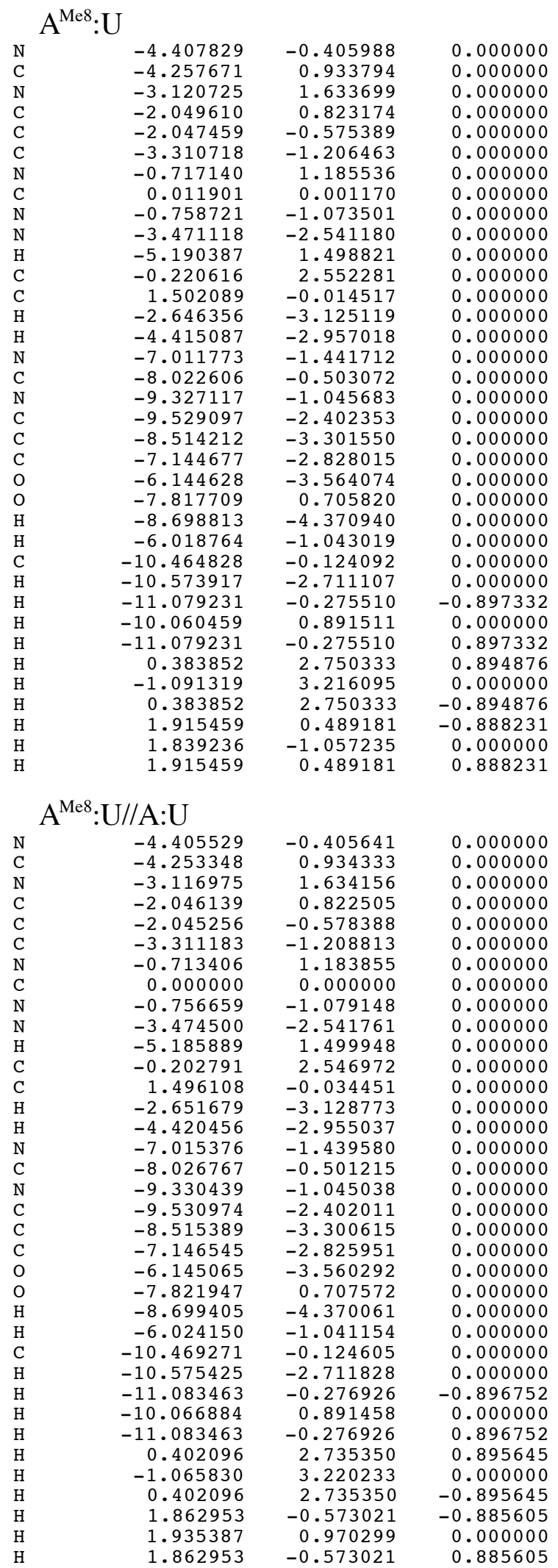


Atomic Cartesian coordinates $(\AA)$ continued

Microsolvated A:T

$\begin{array}{lrrr}\mathrm{N} & -2.775960 & 2.894499 & 0.000000 \\ \mathrm{C} & -1.470004 & 3.223084 & 0.000000 \\ \mathrm{~N} & -0.415681 & 2.403887 & 0.000000 \\ \mathrm{C} & -0.802315 & 1.117987 & 0.000000 \\ \mathrm{C} & -2.112449 & 0.621690 & 0.000000 \\ \mathrm{C} & -3.153010 & 1.587597 & 0.000000 \\ \mathrm{~N} & 0.012440 & 0.001276 & 0.000000 \\ \mathrm{C} & -0.831657 & -1.086853 & 0.000000 \\ \mathrm{~N} & -2.111909 & -0.764972 & 0.000000 \\ \mathrm{~N} & -4.458848 & 1.289868 & 0.000000 \\ \mathrm{H} & -1.268605 & 4.294999 & 0.000000 \\ \mathrm{C} & 1.468653 & 0.014157 & 0.000000 \\ \mathrm{H} & 1.860630 & -0.482858 & -0.895841 \\ \mathrm{H} & 1.785634 & 1.061663 & 0.000000 \\ \mathrm{H} & 1.860630 & -0.482858 & 0.895841 \\ \mathrm{H} & -0.444702 & -2.101017 & 0.000000 \\ \mathrm{H} & -4.763242 & 0.311881 & 0.000000 \\ \mathrm{H} & -5.147892 & 2.053017 & 0.000000 \\ \mathrm{~N} & -4.622314 & 5.013077 & 0.000000 \\ \mathrm{C} & -4.044135 & 6.268228 & 0.000000 \\ \mathrm{~N} & -4.968236 & 7.324622 & 0.000000 \\ \mathrm{C} & -6.323130 & 7.087145 & 0.000000 \\ \mathrm{C} & -6.881097 & 5.846510 & 0.000000 \\ \mathrm{C} & -5.972297 & 4.710408 & 0.000000 \\ \mathrm{O} & -6.340875 & 3.515530 & 0.000000 \\ \mathrm{O} & -2.829682 & 6.444805 & 0.000000 \\ \mathrm{C} & -8.363183 & 5.611442 & 0.000000 \\ \mathrm{H} & -3.931159 & 4.198664 & 0.000000 \\ \mathrm{C} & -4.458240 & 8.697100 & 0.000000 \\ \mathrm{H} & -6.941732 & 7.984737 & 0.000000 \\ \mathrm{H} & -8.673466 & 5.028472 & 0.877971 \\ \mathrm{H} & -8.911677 & 6.561390 & 0.000000 \\ \mathrm{H} & -8.673466 & 5.028472 & -0.877971 \\ \mathrm{H} & -4.798773 & 9.230605 & -0.896574 \\ \mathrm{H} & -3.366998 & 8.639411 & 0.000000 \\ \mathrm{H} & -4.798773 & 9.230605 & 0.896574 \\ \mathrm{O} & -8.945306 & 2.356164 & 0.000000 \\ \mathrm{H} & -8.042101 & 2.746775 & 0.000000 \\ \mathrm{H} & -8.797797 & 1.397796 & 0.000000 \\ & -4.982142 & -1.646317 & 0.000000 \\ \mathrm{H} & -4.362512 & -1.730719 & -0.747496 \\ -4.362512 & -1.730719 & 0.747496\end{array}$

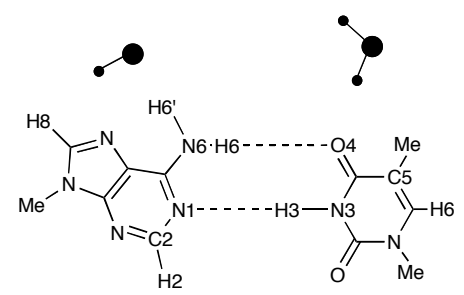

\begin{tabular}{lrrr}
\multicolumn{4}{c}{ Microsolvated A:U } \\
N & -2.775163 & 2.894586 & 0.000000 \\
$\mathrm{C}$ & -1.468877 & 3.221955 & 0.000000 \\
$\mathrm{~N}$ & -0.415337 & 2.402346 & 0.000000 \\
$\mathrm{C}$ & -0.803275 & 1.116821 & 0.000000 \\
$\mathrm{C}$ & -2.113759 & 0.621387 & 0.000000 \\
$\mathrm{C}$ & -3.154334 & 1.587792 & 0.000000 \\
$\mathrm{~N}$ & 0.011089 & 0.000063 & 0.000000 \\
$\mathrm{C}$ & -0.833370 & -1.087738 & 0.000000 \\
$\mathrm{~N}$ & -2.113444 & -0.765249 & 0.000000 \\
$\mathrm{~N}$ & -4.460152 & 1.290714 & 0.000000 \\
$\mathrm{H}$ & -1.266227 & 4.293684 & 0.000000 \\
$\mathrm{C}$ & 1.467327 & 0.013419 & 0.000000 \\
$\mathrm{H}$ & 1.859483 & -0.483352 & -0.895884 \\
$\mathrm{H}$ & 1.783800 & 1.061108 & 0.000000 \\
$\mathrm{H}$ & 1.859483 & -0.483352 & 0.895884 \\
$\mathrm{H}$ & -0.446871 & -2.102082 & 0.000000 \\
$\mathrm{H}$ & -4.765219 & 0.312758 & 0.000000 \\
$\mathrm{H}$ & -5.149697 & 2.053314 & 0.000000 \\
$\mathrm{~N}$ & -4.598622 & 5.024550 & 0.000000 \\
$\mathrm{C}$ & -4.025474 & 6.281830 & 0.000000 \\
$\mathrm{~N}$ & -4.951314 & 7.345410 & 0.000000 \\
$\mathrm{C}$ & -6.302419 & 7.108660 & 0.000000
\end{tabular}




$\begin{array}{lrrr}\mathrm{C} & -6.837533 & 5.862323 & 0.000000 \\ \mathrm{C} & -5.950186 & 4.722809 & 0.000000 \\ \mathrm{O} & -6.323965 & 3.528933 & 0.000000 \\ \mathrm{O} & -2.813241 & 6.463772 & 0.000000 \\ \mathrm{H} & -7.911189 & 5.696381 & 0.000000 \\ \mathrm{H} & -3.907990 & 4.207323 & 0.000000 \\ \mathrm{C} & -4.436818 & 8.717051 & 0.000000 \\ \mathrm{H} & -6.925628 & 8.001972 & 0.000000 \\ \mathrm{H} & -4.776452 & 9.250585 & 0.896745 \\ \mathrm{H} & -3.345813 & 8.656772 & 0.000000 \\ \mathrm{H} & -4.776452 & 9.250585 & -0.896745 \\ \mathrm{O} & -9.120378 & 3.549478 & 0.000000 \\ \mathrm{H} & -8.146566 & 3.386804 & 0.000000 \\ \mathrm{H} & -9.524762 & 2.668650 & 0.000000 \\ \mathrm{O} & -4.978440 & -1.649363 & 0.000000 \\ \mathrm{H} & -4.358619 & -1.732644 & 0.747410 \\ \mathrm{H} & -4.358619 & -1.732644 & -0.747410\end{array}$

$\begin{array}{lrrr} & \text { Microsolvated A:U } & & \\ \mathrm{N} & -5.482770 & 0.551410 & 0.000000 \\ \mathrm{C} & -5.954756 & 1.812212 & 0.000000 \\ \mathrm{~N} & -7.227814 & 2.214095 & 0.000000 \\ \mathrm{C} & -8.065172 & 1.164383 & 0.000000 \\ \mathrm{C} & -7.728874 & -0.195729 & 0.000000 \\ \mathrm{C} & -6.342266 & -0.503386 & 0.000000 \\ \mathrm{~N} & -9.446895 & 1.200653 & 0.000000 \\ \mathrm{C} & -9.862982 & -0.112065 & 0.000000 \\ \mathrm{~N} & -8.870657 & -0.982650 & 0.000000 \\ \mathrm{~N} & -5.843695 & -1.746148 & 0.000000 \\ \mathrm{H} & -5.187350 & 2.587322 & 0.000000 \\ \mathrm{C} & -10.261950 & 2.407362 & 0.000000 \\ \mathrm{H} & -10.893511 & 2.448711 & -0.895900 \\ \mathrm{H} & -9.578410 & 3.262138 & 0.000000 \\ \mathrm{H} & -10.893511 & 2.448711 & 0.895900 \\ \mathrm{H} & -10.917411 & -0.369795 & 0.000000 \\ \mathrm{H} & -6.474780 & -2.552873 & 0.000000 \\ \mathrm{H} & -4.823320 & -1.880132 & 0.000000 \\ \mathrm{~N} & -2.696348 & 0.251020 & 0.000000 \\ \mathrm{C} & -1.978656 & 1.426645 & 0.000000 \\ \mathrm{~N} & -0.573142 & 1.271628 & 0.000000 \\ \mathrm{C} & 0.020149 & 0.020606 & 0.000000 \\ \mathrm{C} & -0.741755 & -1.112350 & 0.000000 \\ \mathrm{C} & -2.177732 & -1.030759 & 0.000000 \\ \mathrm{O} & -2.950732 & -2.017024 & 0.000000 \\ \mathrm{O} & -2.516306 & 2.529814 & 0.000000 \\ \mathrm{H} & -0.282144 & -2.097102 & 0.000000 \\ \mathrm{H} & -3.759074 & 0.361256 & 0.000000 \\ \mathrm{C} & 0.223069 & 2.502370 & 0.000000 \\ \mathrm{C} & 1.522241 & -0.035590 & 0.000000 \\ \mathrm{H} & 1.941674 & 0.463760 & -0.886542 \\ \mathrm{H} & 1.852824 & -1.080206 & 0.000000 \\ \mathrm{H} & 1.941674 & 0.463760 & 0.886542 \\ \mathrm{H} & 0.854911 & 2.557783 & 0.895307 \\ \mathrm{H} & -0.482985 & 3.336085 & 0.000000 \\ \mathrm{H} & 0.854911 & 2.557783 & -0.895307 \\ \mathrm{O} & -1.382464 & -4.330366 & 0.000000 \\ \mathrm{H} & -2.046503 & -3.598588 & 0.000000 \\ \mathrm{H} & -1.906240 & -5.145903 & 0.000000 \\ \mathrm{H} & -7.975118 & -3.842696 & 0.000000 \\ & -8.392775 & -3.376908 & 0.747233 \\ & -8.392775 & -3.376908 & -0.747233\end{array}$


Table S1. NMR shielding constants, bond energy decomposition, and hydrogen bond lengths for the A:T, A:U, A:U//A:T, ${ }^{\mathrm{a}} \mathrm{A}: \mathrm{T} / / \mathrm{A}: \mathrm{U}^{\mathrm{b}}$ and $\mathrm{A}: \mathrm{U}^{\mathrm{Me} 6}$ base pairs (TZ2P basis)

\begin{tabular}{|c|c|c|c|c|c|}
\hline & $\mathrm{A}: \mathrm{T}$ & $\mathrm{A}: \mathrm{U}$ & $\mathrm{A}: \mathrm{T} / / \mathrm{A}: \mathrm{U}$ & A:U//A:T & $\mathrm{A}: \mathrm{U}^{\mathrm{Me} \sigma}$ \\
\hline \multicolumn{6}{|c|}{ NMR shielding (SAOP; in ppm) } \\
\hline N1-A & 17.293 & 17.623 & 17.329 & 17.600 & 17.284 \\
\hline $\mathrm{C} 2-\mathrm{A}$ & 32.438 & 32.538 & 32.454 & 32.526 & 32.499 \\
\hline $\mathrm{H} 3-\mathrm{T} / \mathrm{U}$ & 71.776 & 69.098 & 70.486 & 70.413 & 73.213 \\
\hline $\mathrm{N} 3-\mathrm{T} / \mathrm{U}$ & - & - & - & - & - \\
\hline \multicolumn{6}{|c|}{ Bond energy decomposition $(B P 86 \text {; in } \mathrm{kcal} / \mathrm{mol})^{c}$} \\
\hline$\Delta E_{\mathrm{prep}}$ & 2.16 & 2.25 & 2.29 & 2.59 & 2.24 \\
\hline$\Delta E_{\text {int }}^{\text {prep }}$ & -15.17 & -15.44 & -15.07 & -15.54 & -15.50 \\
\hline$\Delta E_{\text {Pauli }}$ & 38.49 & 38.55 & 38.60 & 38.45 & 39.09 \\
\hline$\Delta V_{\text {elstat }}$ & -31.82 & -32.01 & -31.79 & -32.04 & -32.36 \\
\hline$\Delta E_{o j}^{\text {estat }}$ & -21.84 & -21.98 & -21.88 & -21.95 & -22.22 \\
\hline$\Delta E_{\sigma}^{o l}$ & -20.20 & -20.32 & -20.24 & -20.28 & -20.54 \\
\hline$\Delta E_{\pi}^{\sigma}$ & -1.64 & -1.67 & -1.64 & -1.67 & -1.68 \\
\hline BSSE & 0.67 & 0.68 & 0.69 & 0.68 & 0.71 \\
\hline$\Delta \mathrm{E}_{\text {total }}$ & -12.33 & -12.50 & -12.10 & -12.27 & -12.55 \\
\hline Bond lengths (BP86; in Å) & 2.852 & 2.858 & 2.858 & 2.852 & 2.846 \\
\hline N1-N3 & 2.811 & 2.807 & 2.807 & 2.811 & 2.808 \\
\hline N3-H3 & 1.067 & 1.068 & 1.068 & 1.067 & 1.067 \\
\hline
\end{tabular}

Table S2. NMR shielding constants, bond energy decomposition, and hydrogen bond lengths for the A:T, A:U, A:U//A:T, ${ }^{a} A: T / / A: U^{b}$ and $A: U^{\mathrm{Me} 6}$ base pairs with scalar relativistic $(Z O R A)^{c}$ effects taken into account $(\text { QZ4P basis })^{\mathrm{d}}$

\begin{tabular}{|c|c|c|c|c|c|}
\hline & A:T & $\overline{\mathrm{A}: \mathrm{U}}$ & A:T//A:U & $\mathrm{A}: \mathrm{U} / / \mathrm{A}: \mathrm{T}$ & $\mathrm{A}: \mathrm{U}^{\mathrm{Me} 6}$ \\
\hline \multicolumn{6}{|c|}{ NMR shielding (SAOP; in ppm) } \\
\hline N1-A & 9.447 & 9.779 & 9.489 & 9.766 & 9.441 \\
\hline $\mathrm{C} 2-\mathrm{A}$ & 23.392 & 23.506 & 23.410 & 23.491 & 23.439 \\
\hline $\mathrm{H} 3-\mathrm{T} / \mathrm{U}$ & 63.286 & 61.004 & 61.987 & 62.391 & 65.242 \\
\hline $\mathrm{N} 3-\mathrm{T} / \mathrm{U}$ & 13.878 & 13.784 & 13.781 & 13.880 & 13.916 \\
\hline \multicolumn{6}{|c|}{ Bond energy decomposition $(B P 86 \text {; in } \mathrm{kcal} / \mathrm{mol})^{\mathrm{e}}$} \\
\hline$\Delta E_{\text {nen }}$ & 2.18 & 2.38 & 2.30 & 2.69 & 2.25 \\
\hline$\Delta E_{\text {int }}$ & -15.25 & -15.47 & -15.15 & -15.58 & -15.67 \\
\hline$\Delta E_{P_{\text {iuli }}}$ & 38.85 & 38.93 & 38.95 & 38.83 & 39.47 \\
\hline$\Delta V_{\text {elsalt }}$ & -31.54 & -31.73 & -31.50 & -31.77 & -32.09 \\
\hline$\Delta E_{o j}^{\text {estat }}$ & -22.56 & -22.67 & -22.60 & -22.64 & -23.05 \\
\hline$\Delta E_{\sigma}^{o i}$ & -20.73 & -20.81 & -20.77 & -20.78 & -21.16 \\
\hline$\Delta E_{\pi}^{\sigma}$ & -1.83 & -1.86 & -1.83 & -1.86 & -1.89 \\
\hline BSSE & 0.72 & 0.65 & 0.68 & 0.65 & 0.78 \\
\hline$\Delta \mathrm{E}_{\text {total }}$ & -12.35 & -12.44 & -12.16 & -12.24 & -12.64 \\
\hline
\end{tabular}


Table S3. NMR shielding constants (ppm), bond energy decomposition ( $\mathrm{kcal} / \mathrm{mol})$ and hydrogen bond lengths $(\AA)$ for the $A: U^{M e 6}, A: U^{M e 6} / / A: U, A^{\mathrm{Me} 8}: U, A^{\mathrm{Me} 8}: U / / A: U$, and $H 3$ displaced A:T and A:U base pairs (TZ2P basis)

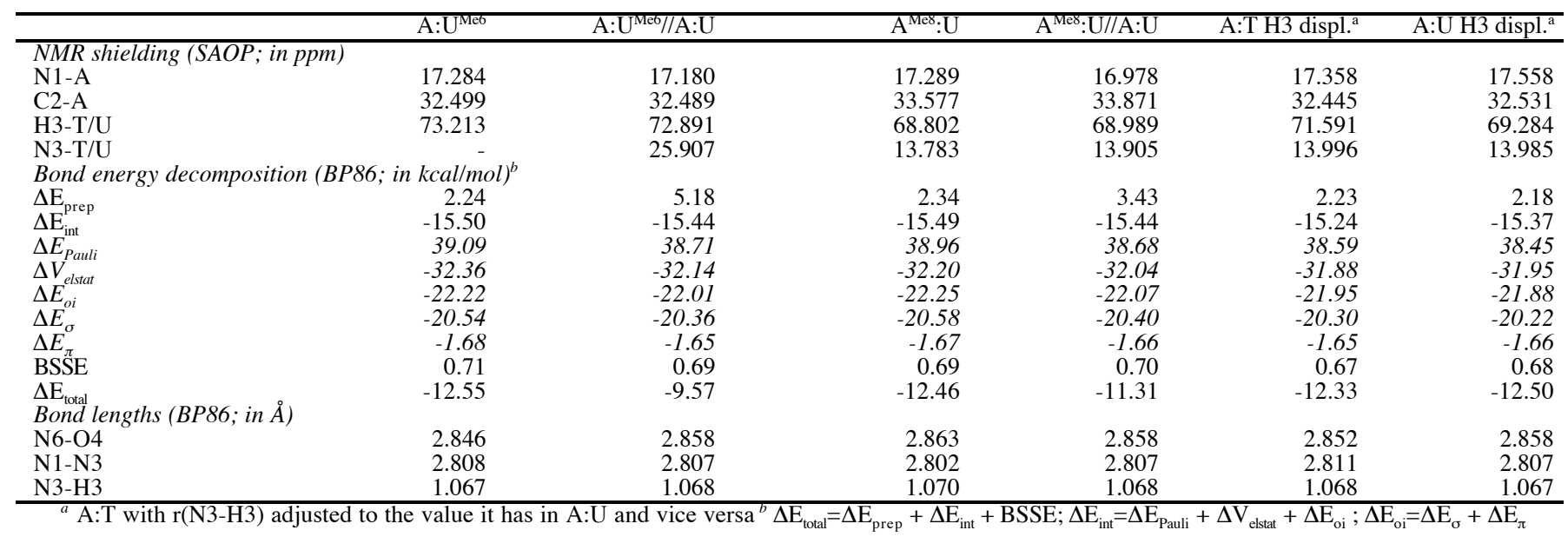

Table S4. Computational results for the A:T, A:U, A:T//A:U, A:U//A:T, A: $\mathrm{U}^{\mathrm{Me} 6}$ base pairs, with two water molecules at the available major-groove positions, fully geometry-optimized (see illustration below, left), computed at SAOP/TZ2P, and without solvent (see illustration below, right) computed at the $a b$ initio Hartree-Fock level with a mixed basis set ${ }^{\mathrm{a}, \mathrm{b}}$

\begin{tabular}{|c|c|c|c|c|c|}
\hline & A:T & $\mathrm{A}: \mathrm{U}$ & A:T//A:U & A:U//A:T & A: $U^{\mathrm{Me}}$ \\
\hline $\begin{array}{l}\text { Microsolvated base pairs } \\
\text { C2-A NMR shielding (in ppm) }\end{array}$ & 31.579 & 31.735 & 31.618 & 31.706 & 31.695 \\
\hline $\begin{array}{l}\text { HF/mixedbasis }{ }^{b} \\
\mathrm{C}^{b}-\mathrm{A} \text { NMR shielding (in ppm) } \\
\left.\Delta \mathrm{E}_{\text {total }} \text { (in } \mathrm{kcal} / \mathrm{mol}\right)^{\mathrm{c}}\end{array}$ & $\begin{array}{r}-21.271 \\
-7.44\end{array}$ & $\begin{array}{r}-31.385 \\
-7.63\end{array}$ & $\begin{array}{r}-21.116 \\
-7.04\end{array}$ & $\begin{array}{r}-31.481 \\
-7.55\end{array}$ & $\begin{array}{r}-18.606 \\
-7.58\end{array}$ \\
\hline
\end{tabular}
(N1(A), C2(A), H2(A), N6(A), H6(A), N3(T/U), H3(T/U), C4(T/U), O4(T/U), C5(T/U), H5/C(T/U)), 6-31G on others; limitation to 255 orbitals in NMR code. ${ }^{c} \Delta \mathrm{E}_{\text {total }}=\Delta \mathrm{E}_{\text {prep }}+\Delta \mathrm{E}_{\text {int }}+\mathrm{BSSE}$.

solvents

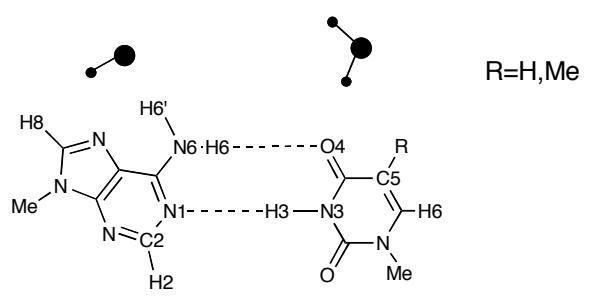

model system used in HF calculations

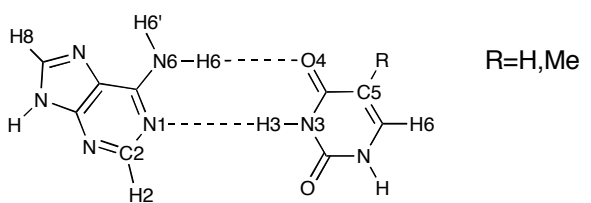


Figure S1. Influence of basis set size cq. DFT functional on C2-A NMR shielding

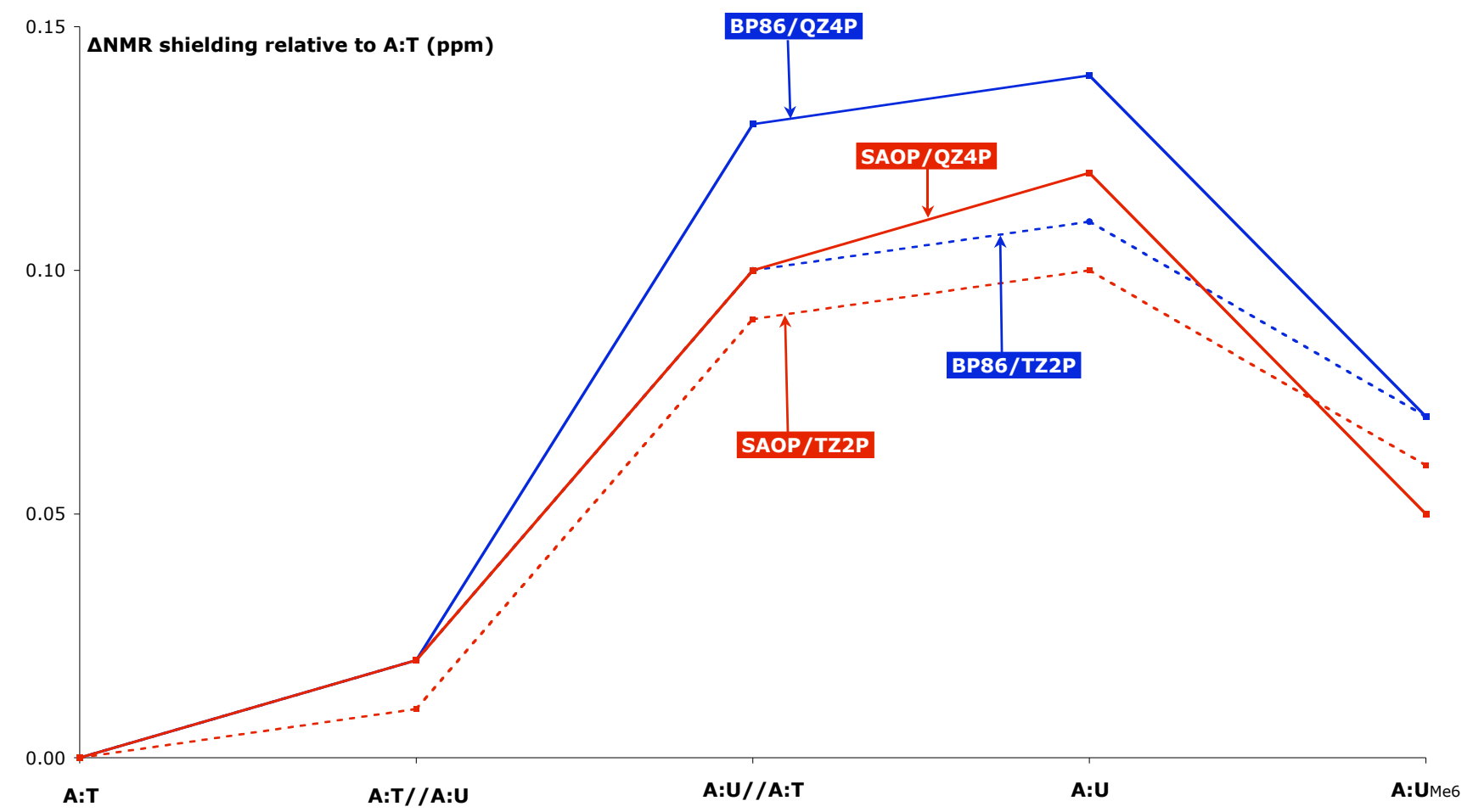

\section{Definition of coordinate axes for variations of base pair geometry}

Interaction energy and C2(A) NMR shielding constant as function of the base pair geometry parameters is plotted in Figures S2 through S5, using the following definition of the $\mathrm{x}$ - and $\mathrm{y}$-axis:

- the $\mathrm{X}$-axis goes through the adenine $\mathrm{C} 6$ and thymine/uracil $\mathrm{C} 4$ atoms

- the $y$-axis is perpendicular to the $x$-axis, exactly in the middle of these two atoms

\section{Adenine-Thymine/Uracil}

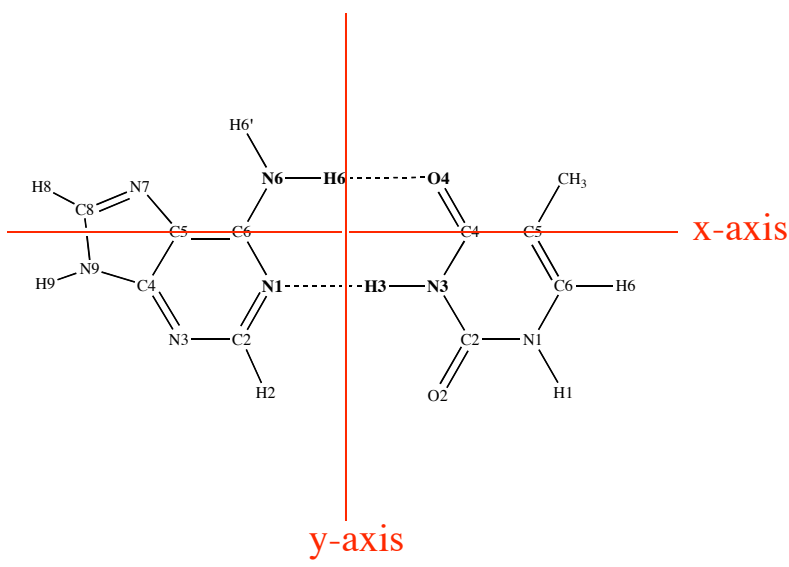


Figure S2. Variations of base pair geometry: Stretch

\section{Stretch: $\Delta$ Eint}

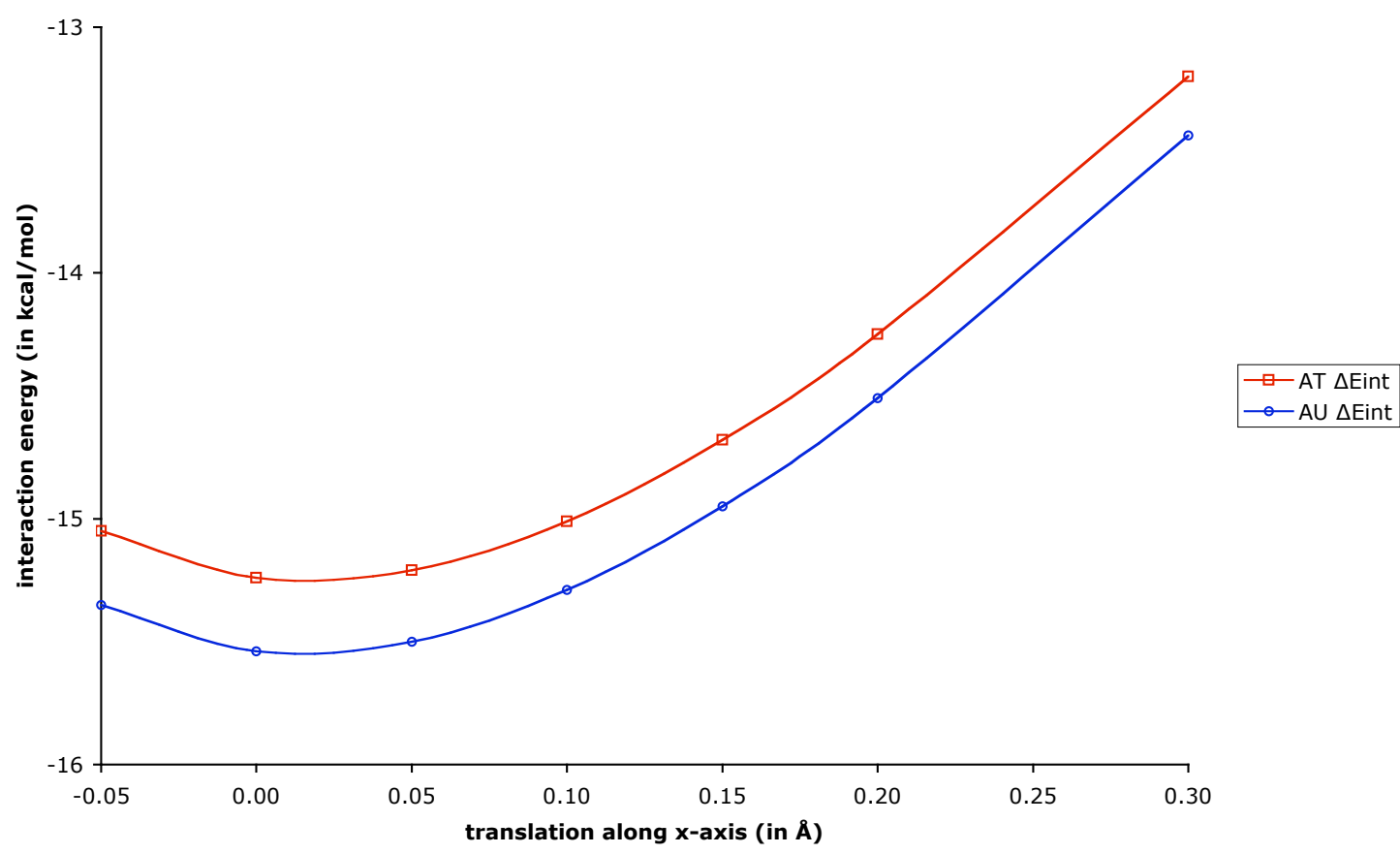

Stretch: C2 NMR shielding

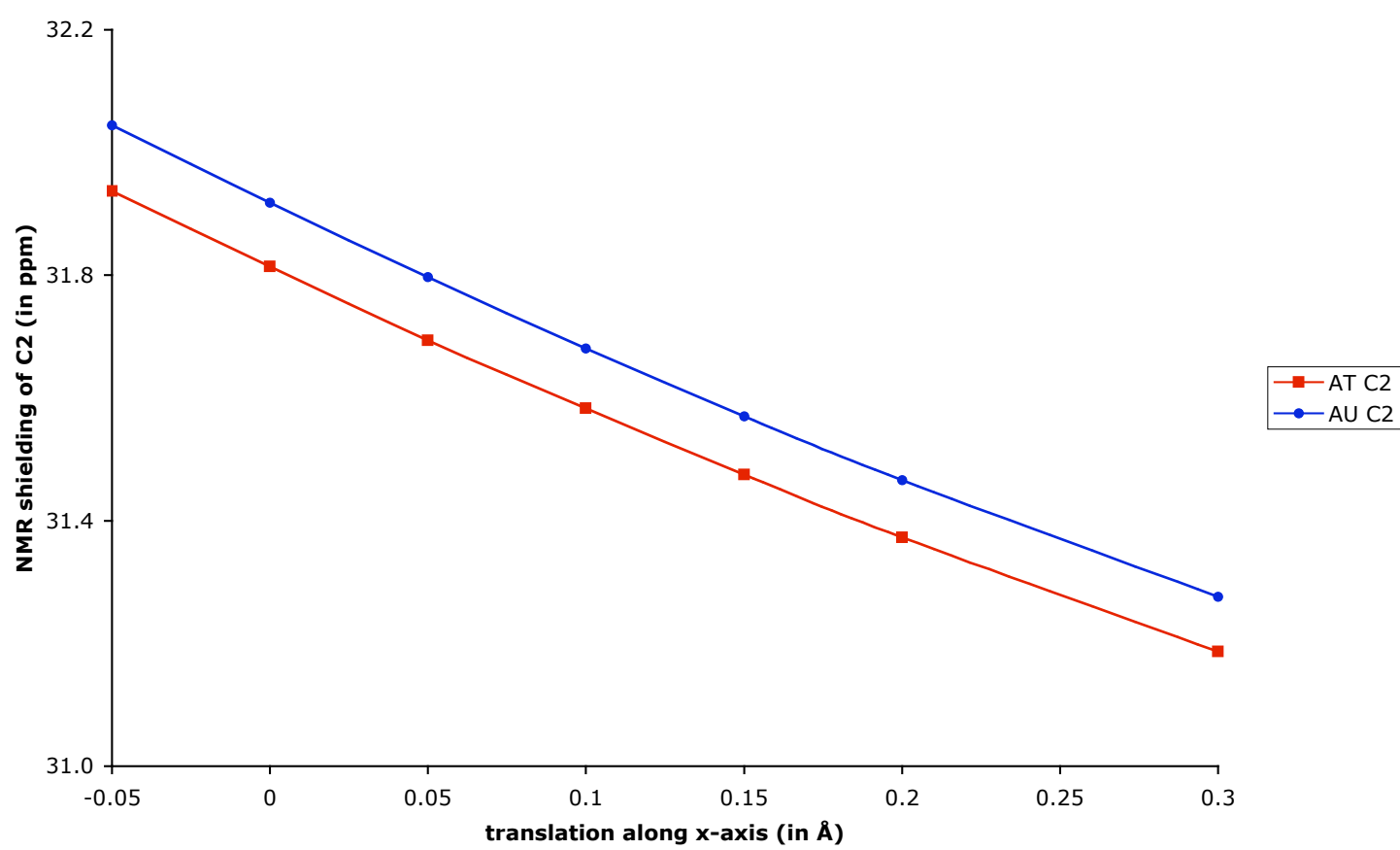


Figure S3. Variations of base pair geometry: Shear

\section{Shear: $\Delta$ Eint}

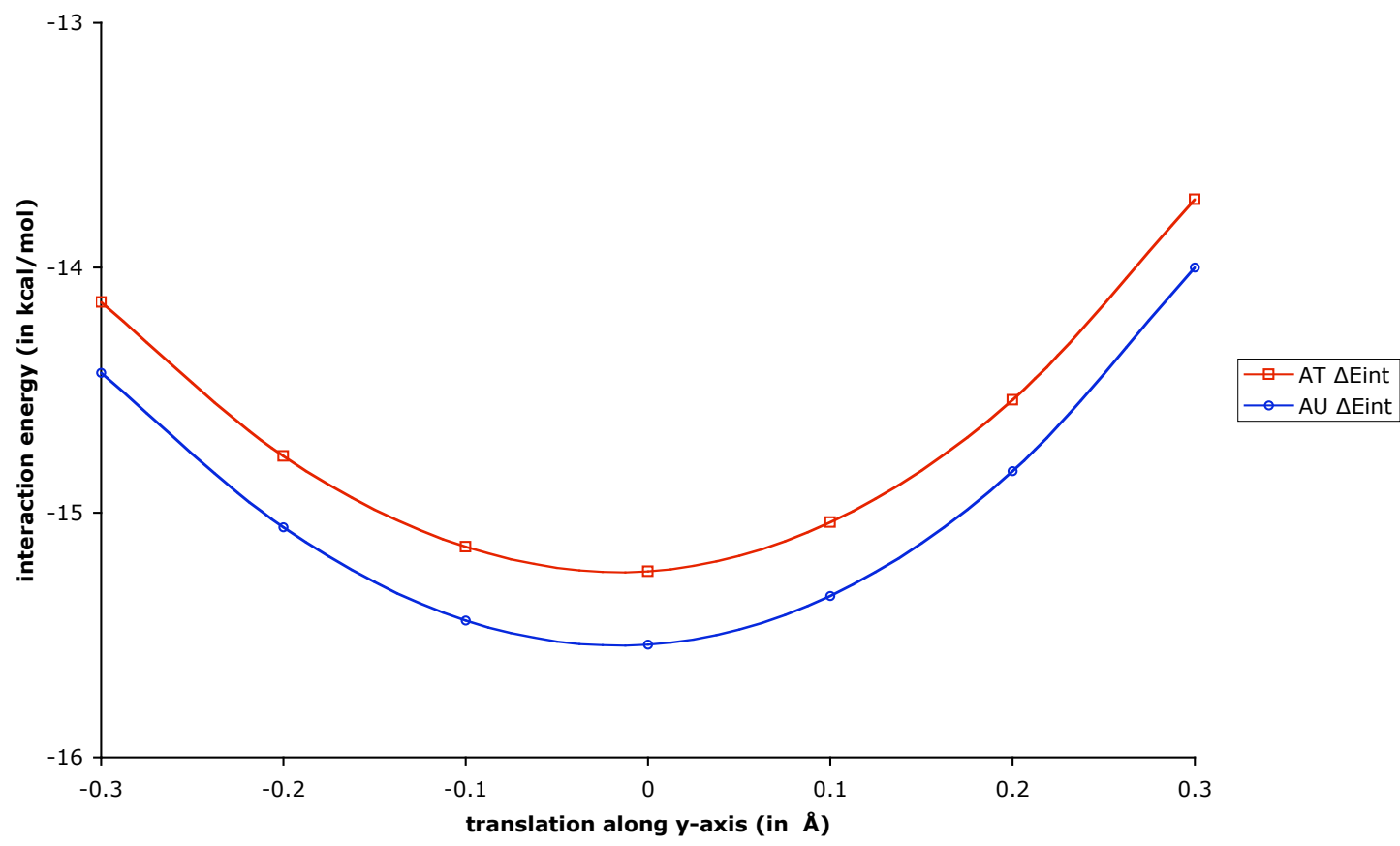

Shear: C2 NMR shielding

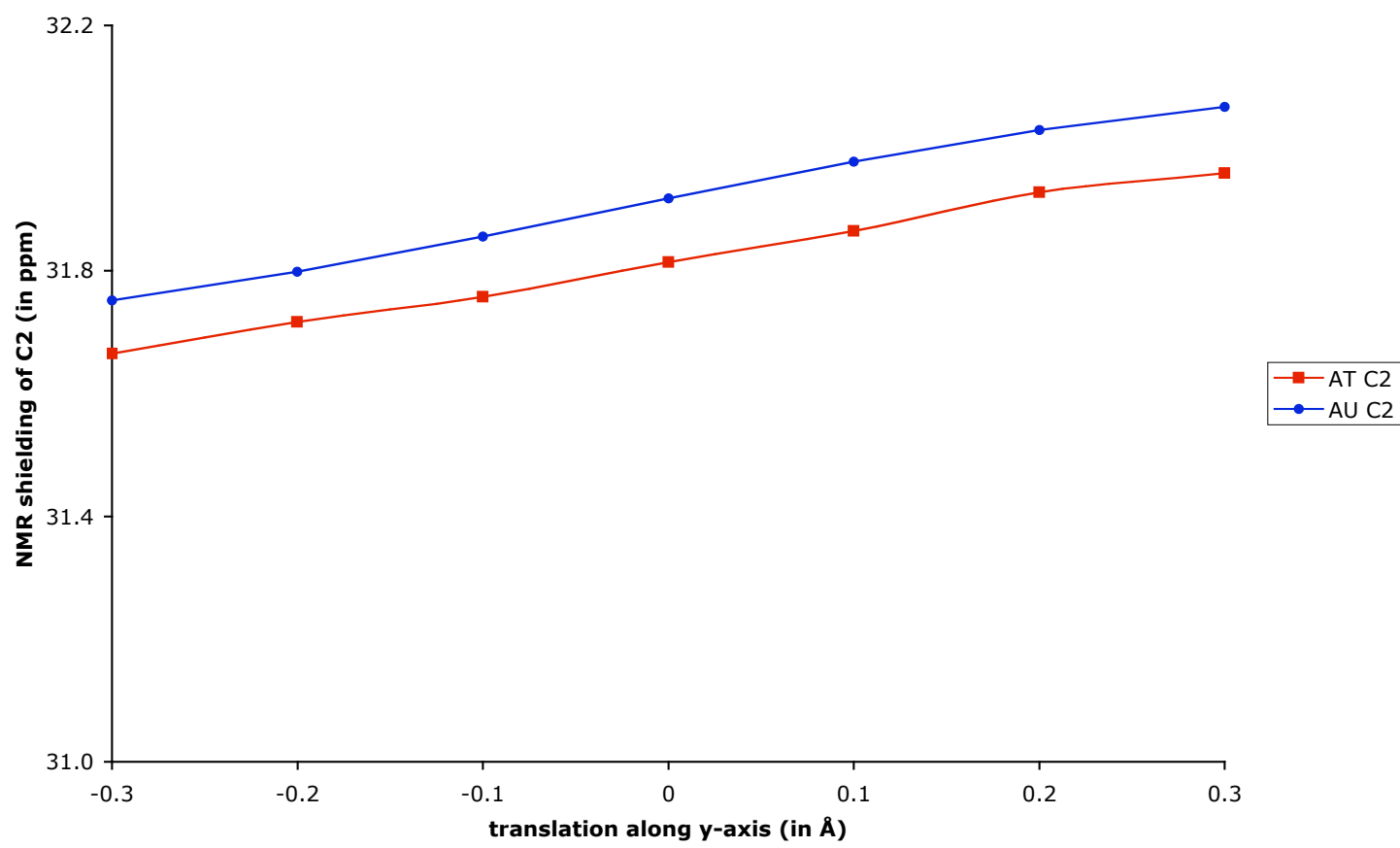


Figure S4. Variations of base pair geometry: Propellor Twist

\section{Propeller Twist: $\Delta$ Eint}

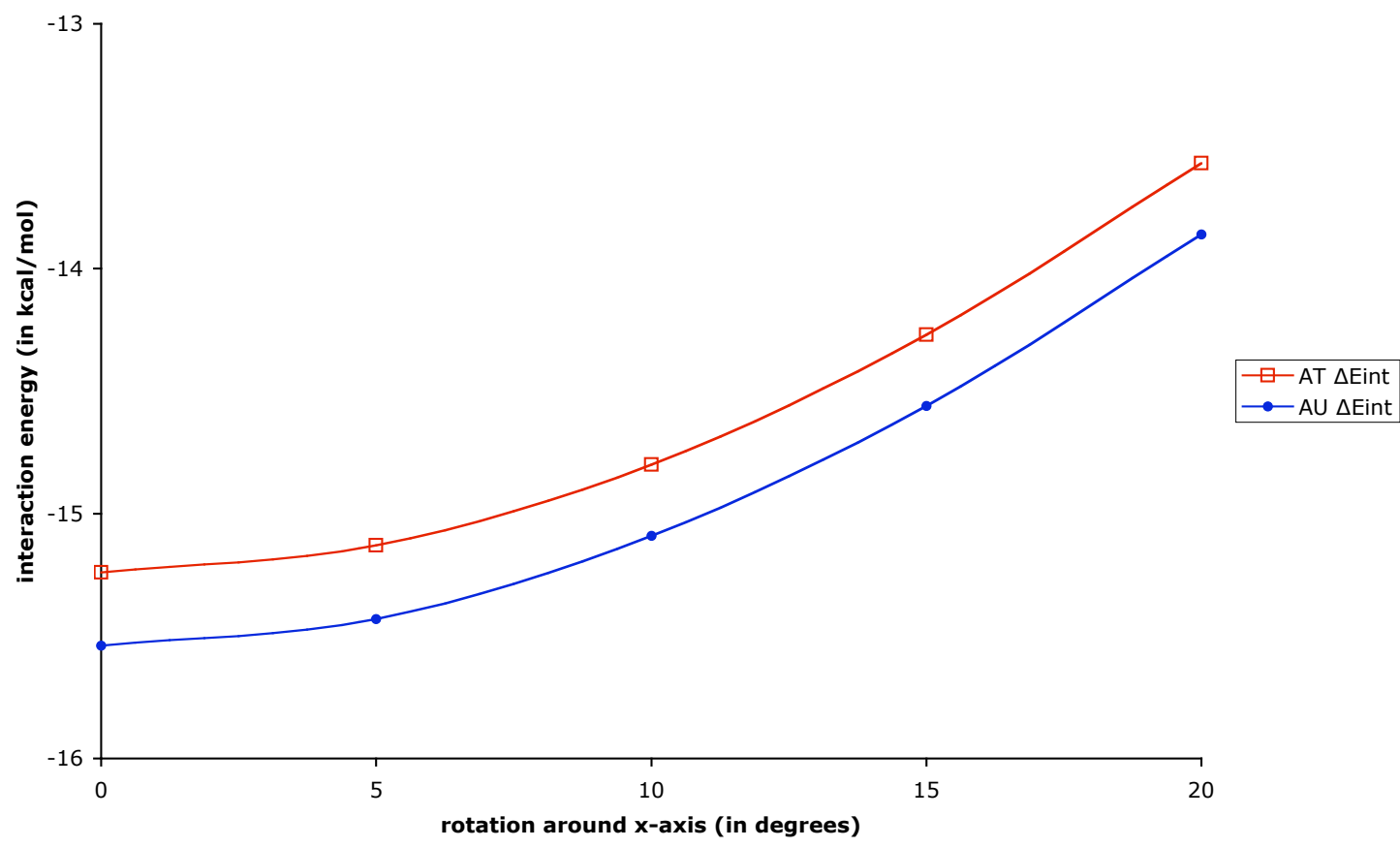

Propeller Twist: C2 NMR shielding

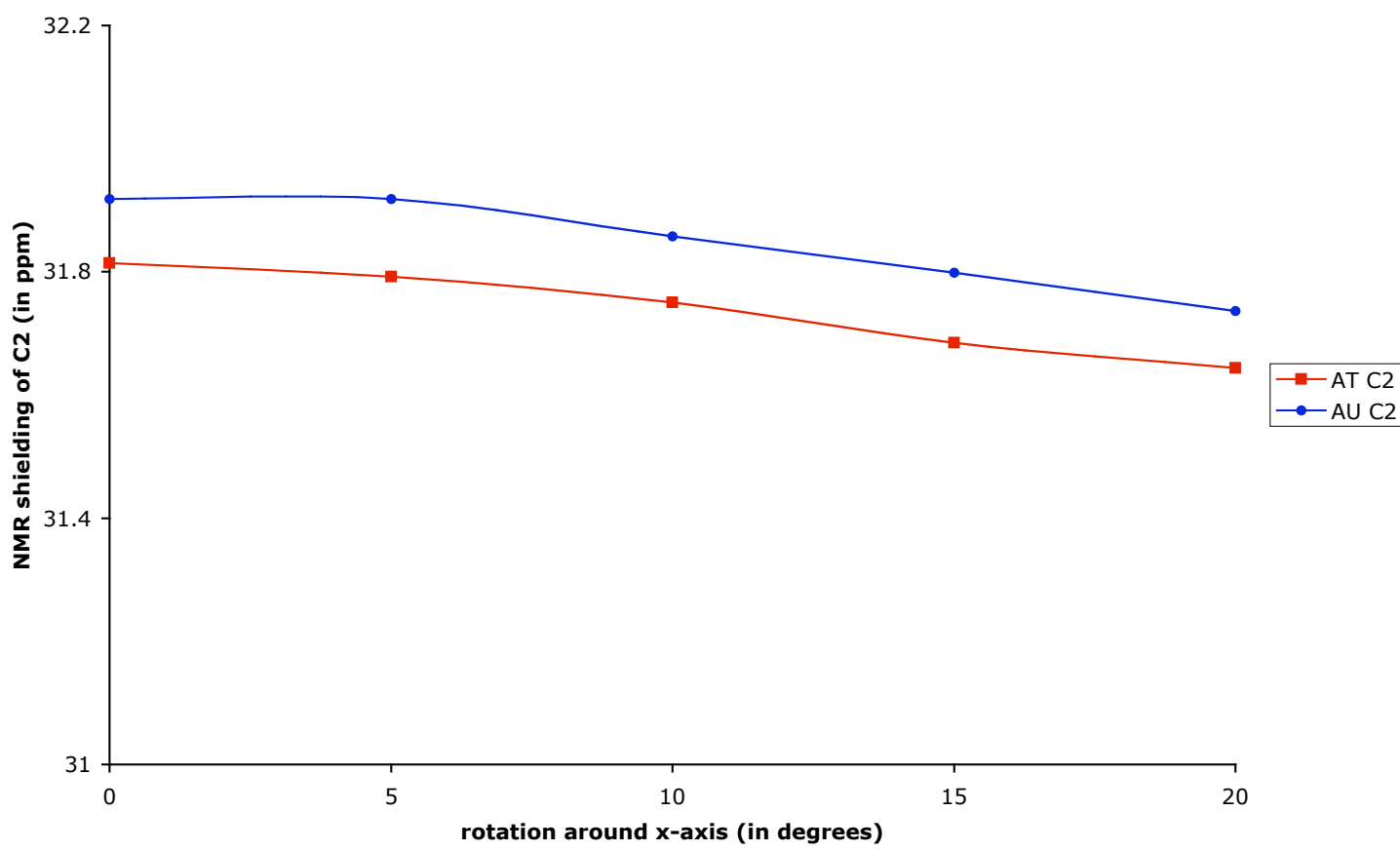


Figure S5. Variations of base pair geometry: Buckle

\section{Buckle: $\Delta$ Eint}

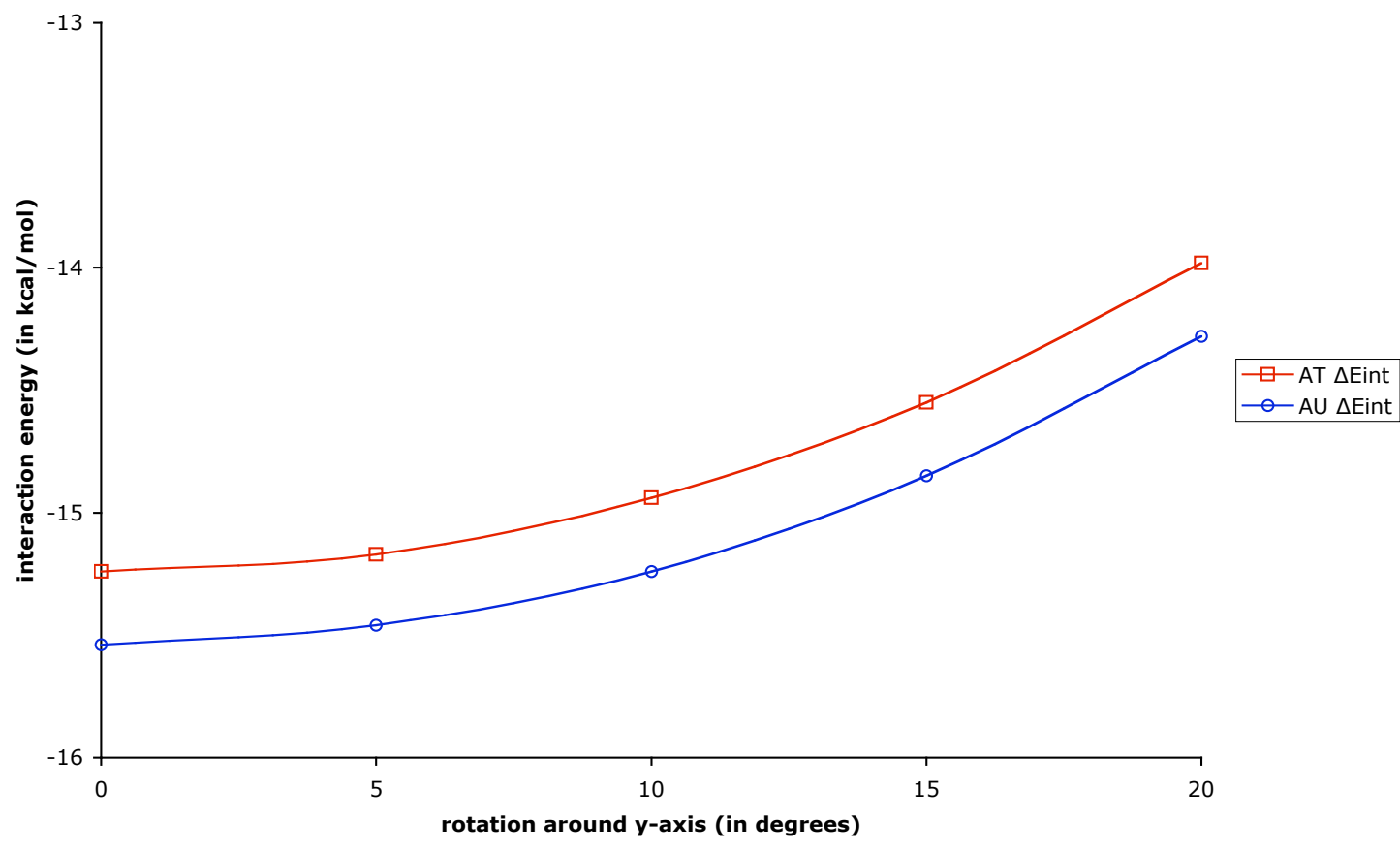

Buckle: C2 NMR shielding

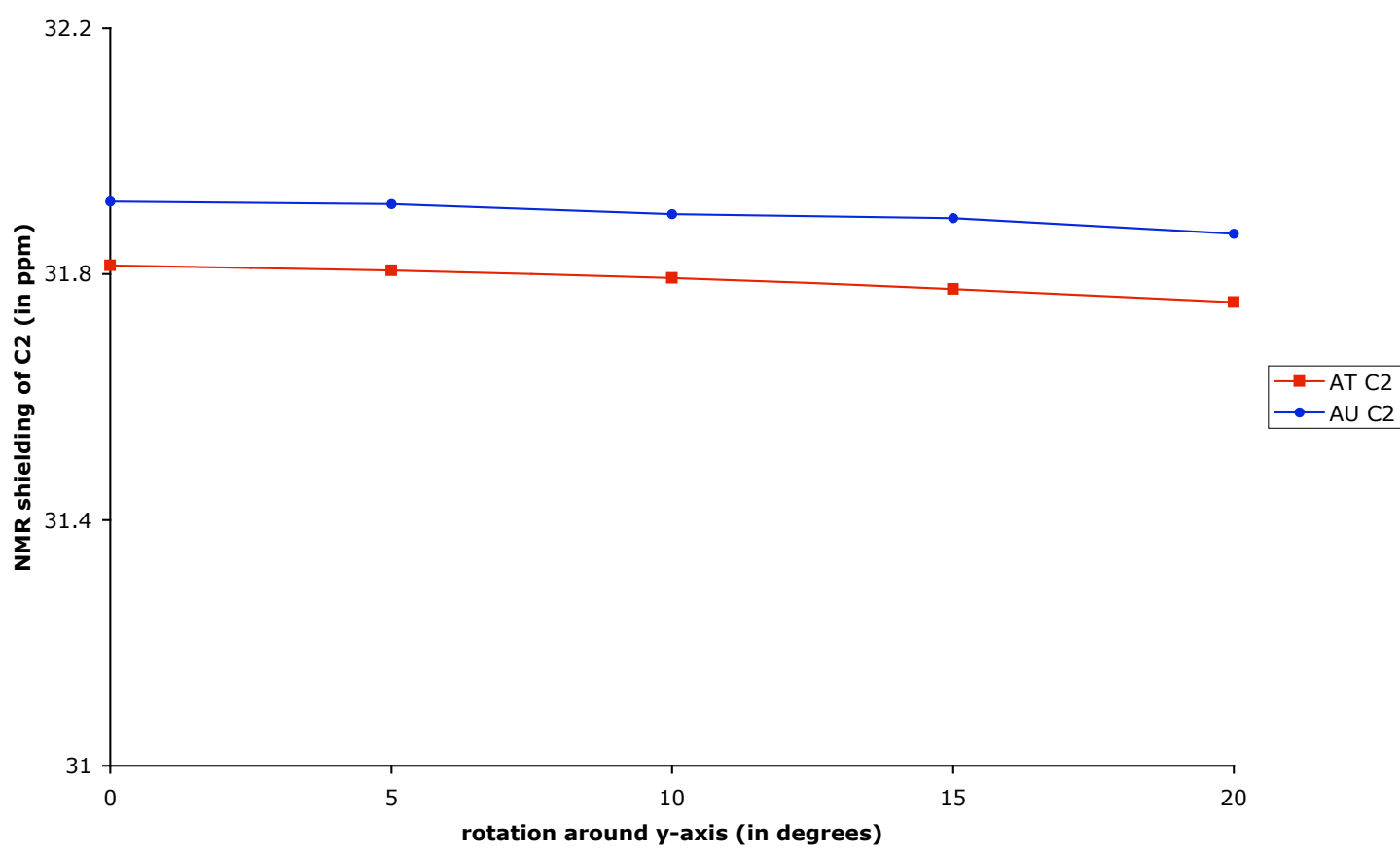

